\title{
Three-Dimensional Simulation of Backward Raman Amplification
}

\author{
A. A. Balakin, G. M. Fraiman, and Nathaniel J. Fisch
}

\begin{abstract}
Three-dimensional (3-D) simulations for the backward Raman amplification (BRA) are presented. The images illustrate the effects of pump depletion, pulse diffraction, nonhomogeneous plasma density, and plasma ionization.
\end{abstract}

Index Terms-Laser amplifier, plasma applications, Raman lasers.

$\mathbf{T}$ HE amplification by resonant Raman backscattering in plasmas currently represents one of the most promising ways of generating ultra-intense short laser pulses. Compared to the conventional technique of chirped-pulse amplification, in principle unfocused output intensity can be $10^{4}-10^{5}$ times higher [1], [2]. In achieving these amplification effects, two optical systems may be utilized: one system for handling high power and fluence, and a second focusing system handling only low power [3].

In describing the propagation of both the laser pump and the counterpropagating low power seed pulse, it is important to take into account the three-dimensional (3-D) effects of pulse diffraction, the noncoaxial pump and seed pulses, refraction on plasma inhomogeneity, and spreading due to ionization. These effects cannot be fully described using two-dimensional (2-D) or axial symmetric approaches.

Here, we present the results of the numerical simulations of the seed pulse Raman amplification. The numerical scheme involves solving the three-dimensional equations describing the three-wave interaction process [1], []. The plasma density is determined using a field ionization model, since the time of the impact ionization is small in comparison to the characteristic time of the backward Raman amplification (BRA) (several picoseconds).

For these calculations, the pump wave amplitude is $a_{0}=$ 0.006 , with Gaussian transverse profile with width $0.15 \mathrm{~cm}$, and the frequency of the pump is $\omega_{0}=2 \times 10^{15} \mathrm{sec}^{-1}$. For

Manuscript received July 19 2004; revised. This work was supported in part by the Russian Foundation for Basic Research (RFBR) under Grants 02-0217275 and 02-02-17277, in part by the U.S. Department of Energy under Contract DE-AC02-76 CHO3073, in part by the U.S. Defense Advanced Research Projects Agency (DARPA), and in part by the National Nuclear Security Administration under the Stewardship Science Academic Alliances program through DOE Research Grant DE-FG52-04NA00139.

A. A. Balakin and G. M. Fraiman are with the Institute of Applied Physics Russian Academy of Sciences, Nizhnii Novgorod, Russia 603950 (e-mail: balakin@appl.sci-nnov.ru; fraiman@appl.sci-nnov.ru).

N. J. Fisch is with the Department of Astrophysical Sciences and the Plasma Physics Laboratory, Princeton University, Princeton, NJ 08543 USA (e-mail: fisch@princeton.edu).

Digital Object Identifier 10.1109/TPS.2005.845905

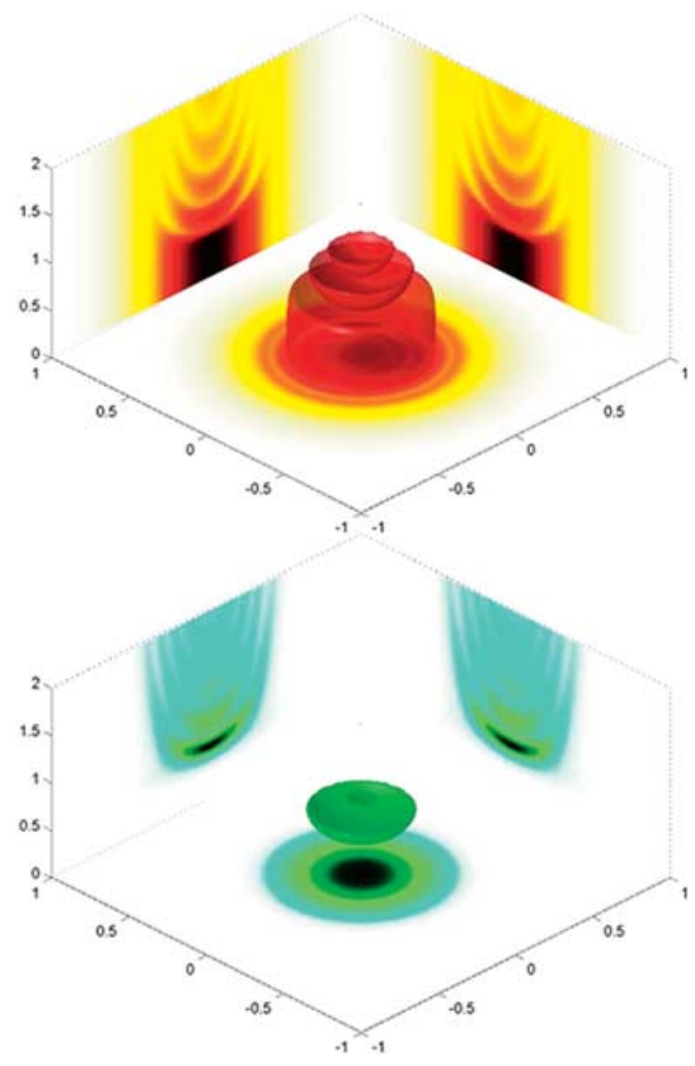

Fig. 1. (Upper) pump and (lower) seed profile for diffractionless case. Transverse coordinates are normalized to $30 \mathrm{~cm}$; longitudinal coordinate is normalized to $c / \gamma$.

these parameters, the linear e-folding length for the monochromatic pump backscattering instability is $\gamma=a_{0} \sqrt{\omega_{0} \omega_{p} / 2}$, or $c / \gamma=130 \mu \mathrm{m}$. We simulated a plasma length of $7 \mathrm{~mm}$ or $55 c / \gamma$. The initial seed pulse had the same transverse Gaussian profile as the pump, but half the amplitude and a duration of 40 fs (12 $\mu \mathrm{m}$ in length).

The numerical simulation was performed using the hydrodynamic code "MBRS" [4]. The code is fully parallelized for using in computer cluster so that the calculation time of typical 3-D variant with grid size $128 \times 128 \times 2750$ points is about $32 \mathrm{~h}$ on 15 processes AMD Athlon $1.7 \mathrm{GHz}$.

Fig. 1 shows the well-known $\pi$-pulse solution for wide pulses in homogeneous medium. The pulse diffraction is negligible for this case. The central surface is an isosurface of constant amplitude value, which is equal to half the maximum pulse amplitude. The figure also plots the density, through a projection onto the corresponding edges. The modification of the $\pi$-pulse solution in the presence of strong diffraction is shown on Fig. 2. The 

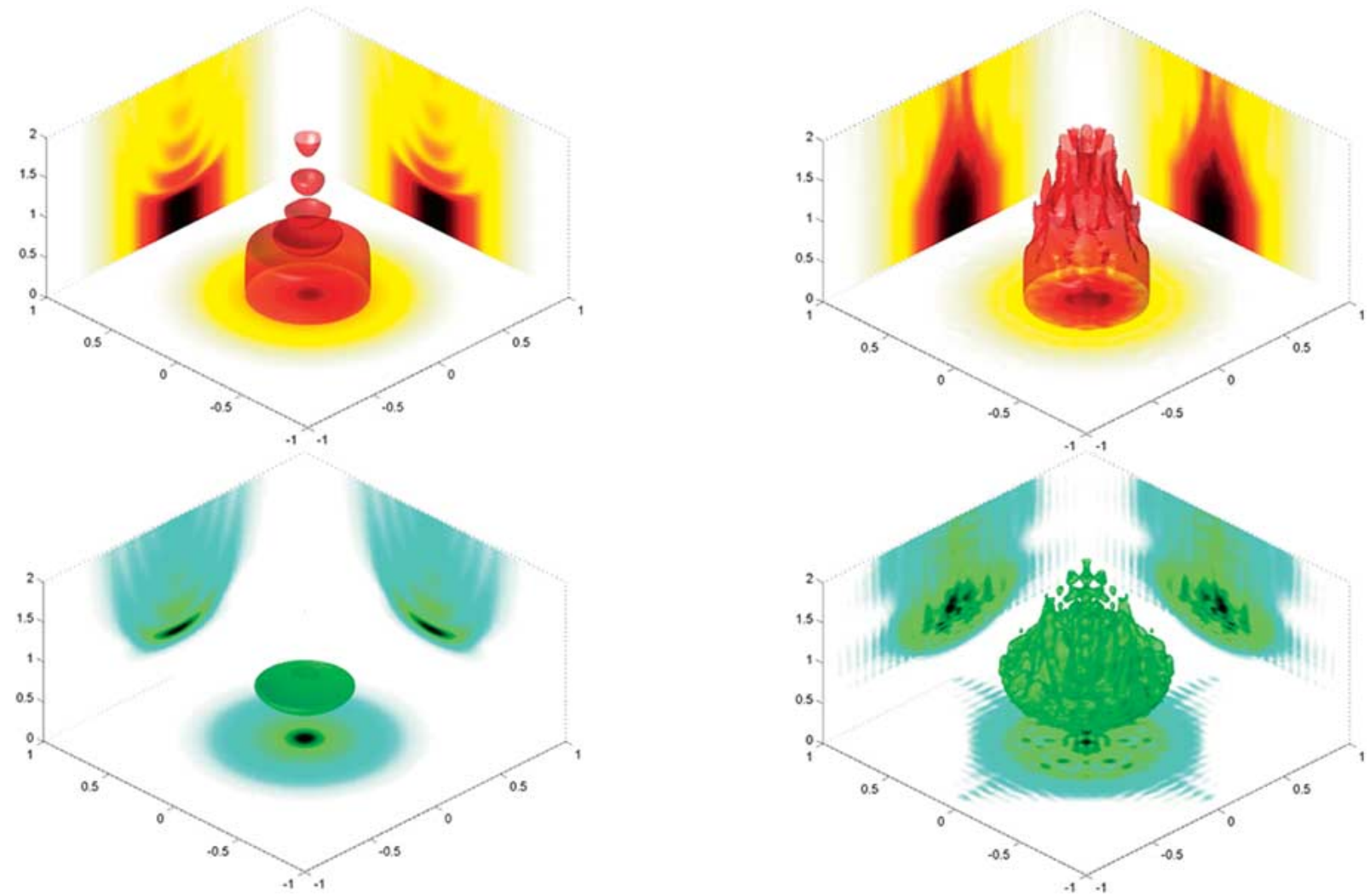

Fig. 2. (Top ) Pump and (bottom) seed profile for case of narrow pump. Transverse coordinates are normalized to $0.3 \mathrm{~cm}$; longitudinal coordinate is normalized to $c / \gamma$.

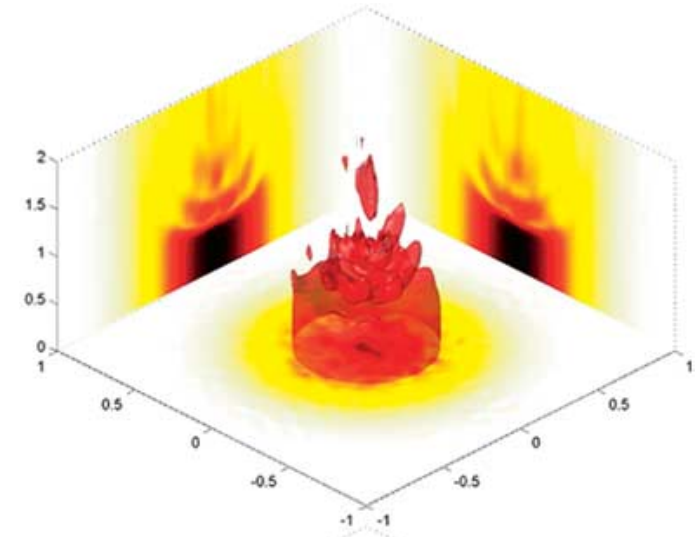

Fig. 4. (Top) pump and (bottom) seed profile for case of amplification in Helium plasma, so that additional ionization is available. Transverse coordinates are normalized to $0.3 \mathrm{~cm}$; longitudinal coordinate is normalized to $c / \gamma$.

pulse width is $150 \mu \mathrm{m}$. One can see the spreading of the pulses on the density plots and the modification of the longitudinal profile. The amplification in inhomogeneous plasma is shown in Fig. 3. One can see the undulating amplitude profile of the seed pulse. Nevertheless, the seed maintains good focusability, since the density perturbations are small (order of 1\%). Amplification in Helium plasma is shown in Fig. 4. Note the formation of "medusa-like" structure of seed. This structure appears due to the breaking of the amplification and the limitation of seed amplitude growth. Also, the pump depletion in this regime is suppressed, showing a lengthy "tongue-like" pump profile. The transverse spikes are formed due to creation of lengthy strips of newly ionized plasma.

\section{REFERENCES}

[1] V. M. Malkin, G. Shvets, and N. J. Fisch, Phys. Rev. Lett., vol. 82, p. 4448, 1999.

[2] V. M. Malkin, G. Shvets, and N. J. Fisch, Phys. Plasmas, vol. 7, p. 2232, 2000.

Fig. 3. (Top) pump and (bottom) seed profile for case of propagation in inhomogeneous plasma. Transverse coordinates are normalized to $0.3 \mathrm{~cm}$; longitudinal coordinate is normalized to $c / \gamma$.

[3] N. J. Fisch and V. M. Malkin, Phys. Plasma, vol. 10, p. 2056, 2003.

[4] A. A. Balakin, G. M. Fraiman, N. J. Fisch, and V. M. Malkin, Phys. Plasma, vol. 10, p. 4856, 2003 